\title{
Maxillary sinus lift performed using ultrasound. Evaluation of 21 patients
}

\author{
Cristina Sánchez-Recio ${ }^{1}$, Maria Peñarrocha-Diago ${ }^{2}$, Miguel Peñarrocha-Diago ${ }^{3}$, David Peñarrocha-Oltra ${ }^{4}$ \\ ${ }^{1}$ Master of Oral Surgery and Implantology. Valencia University Medical and Dental School. Valencia, Spain \\ ${ }^{2}$ Assistant professor of Oral Surgery. University Medical and Dental School. Professor of Master of Oral Surgery and Implanto- \\ logy. Valencia University Medical and Dental School. Valencia, Spain \\ ${ }^{3}$ Professor of Oral Surgery. Director of Master of Oral Surgery and Implantology. Valencia University Medical and Dental \\ School. Valencia, Spain \\ ${ }^{4}$ Resident of the Master of Oral Surgery and Implantology. Valencia University Medical and Dental School. Valencia, Spain
}

Correspondence:

Cirugía Bucal. Clínica Odontológica.

Gascó Oliag 1

46021-Valencia. Spain

Maria.Penarrocha@uv.es

Received: 30/06/2008

Accepted: 02/08/2009

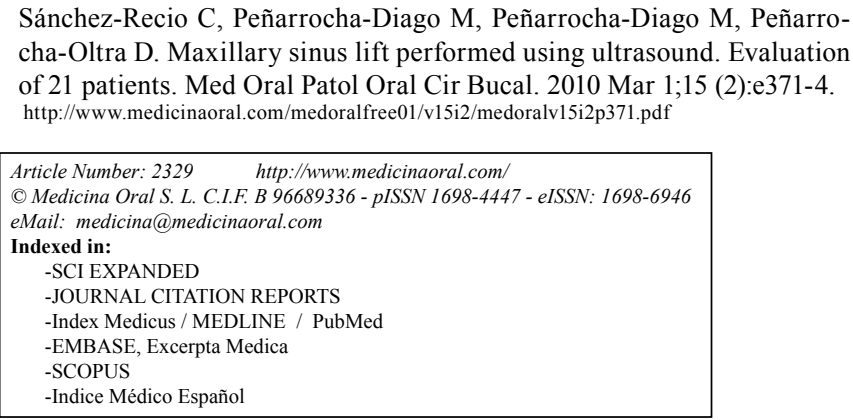

\begin{abstract}
Purpose: The aim of the present study was to evaluate the sinus membrane perforations that occurred during a sinus lift procedure using the ultrasound technique, and to evaluate the bone gain obtained. Materials and methods: In 21 patients, 26 sinus lifts were performed using ultrasound and filled with bone graft material. The bone height and the bone gain obtained were observed in postoperative orthopantomographs, correcting for previous distortion. Results: Of the 26 maxillary sinus lifts, 4 Schneiderian membrane perforations were observed. The average bone height prior to the intervention was $3.5 \mathrm{~mm}$ (scale $0.6-8.7 \mathrm{~mm}$ ) and the average postsurgical bone height was $10.8 \mathrm{~mm}$ (scale $7.5-15.6 \mathrm{~mm}$ ). An average bone gain of $7.2 \mathrm{~mm}$ was observed (range $2.5-11.7 \mathrm{~mm}$ ). Conclusions: Based on the results of this study, during ultrasound sinus lift, few Schneiderian membrane perforations occurred and all were small .
\end{abstract}

Key words: Ultrasound device maxillary surgery, sinus augmentation.

\section{Introduction}

Sinus lift is generally considered to be a safe surgical procedure with a low prevalence of complications $(1,2)$. It is a relatively simple and predictable surgical technique (3).

However, surgical procedures involving bone grafting and implants in the maxillary sinus have potential complications that can be specific or non-specific for these procedures. For sinus graft, perforations of the Schneiderian membrane are the main intraoperative complication occurring in $7 \%$ to $35 \%$ of the procedures (4). Gen- erally, this perforation occurs when making the osseous window to access the sinus using a round diamond drill during the rotary ostectomy stage (3).

To reduce this complication, the creation of a vestibular ostectomy using an odontologic ultrasonic generator is proposed (3) since soft tissue cannot be damaged with this method (5).

Several studies exist on sinus lift made by the ultrasound technique, some of which are preliminary studies describing the technique (3). Subsequently, Vercellotti et al. (6) studies a series of 21 sinus lift with 5\% mem- 
brane perforations; and in 2005 Stübinger et al. (5) presented four isolated cases.

The purpose of this study was to evaluate the sinus membrane perforations occurring during the sinus lift procedure using the ultrasound technique, and to calculate the bone gain obtained.

\section{Material and Methods}

From April 2004 to February 2008, 21 patients were treated with 26 sinus floor augmentation procedures with ultrasound.

The inclusion criteria were: patients with a residual alveolar crest of less than $6 \mathrm{~mm}$ in the posterior atrophic maxillary, cases in which sinus floor augmentation for at least one of the implants was indicated, and patients without systemic disease.

Patients with uncontrolled systemic disease, ongoing chemo- or radiotherapy or a history of maxillary sinus disease were excluded.

All patients were informed of the risks of the maxillary sinus lift and the possible complications; written informed consent was obtained.

In smokers, the numbers of cigarettes per day was recorded, although smoking was not considered a contraindication for treatment.

Before treatment, a clinical and radiographic examination was made in all patients, using panoramic radiography (OPT) (Orthopantomograph OP100. Instrumentarium Imaging, Tuusula, Finlandia).

A total of 48 implants were placed: 44 Defcon ${ }^{\circledR}$ (Impladent; Senmenat, Barcelona, Spain), and 4 Straumann ${ }^{\circledR}$ (Straumann, Basel, Switzerland).

As filling material, either autologous bone, Bio-Oss (Geistlich Pharma AG. Switzerland) or a mixture of both at $50 \%$ was used. The autologous bone was collected from the retromolar trigone or from the maxillary tuberosity.

Surgical procedure

All operations were carried out by the same surgeon (MPD). Surgery was performed under local anesthesia (Ultracain DS forte, Aventis Pharma, Zurich, Switzerland). A sulcular incision was made with two additional releasing incisions, one in the mesial part of the first premolar and the other in the distal part of the first molar. The full-thickness mucoperiosteal flap was lifted upward to expose the complete lateral wall of the maxilla.

Active tips ES008 and ES008A of the Surgysonic ${ }^{\circledR}$ system (Esacrom, Imola, Italy) were used to form an osseous window 1-2 $\mathrm{cm}$ in diameter, with copious sterile saline irrigation (Fig. 1A).

The Schneiderian membrane was separated using tips ES003A and ES003B, and was raised with the tip ES004. Care was taken not to perforate the sinus membrane (Fig. 1B). Schneiderian membrane perforations were not considered a reason to abort the planned augmentation procedure. Depending on the extent of the perforation, various treatment options were performed using different techniques and materials (classification described by Hernández-Alfaro et al.) (7). All perforations were smaller than $5 \mathrm{~mm}$ and patched with a collagen membrane (Lyostypt, B. Braun, Aesculap, Tuttlingen, Germany) in direct contact with the Schneiderian membrane.

The space obtained with the sinus lift was filled with graft material (autologous bone, Bio-Oss or a mixture of both at 50\%) (Fig. 1C). The amount of graft material at each site varied according to the extent of maxillary bone resorption and sinus anatomy.

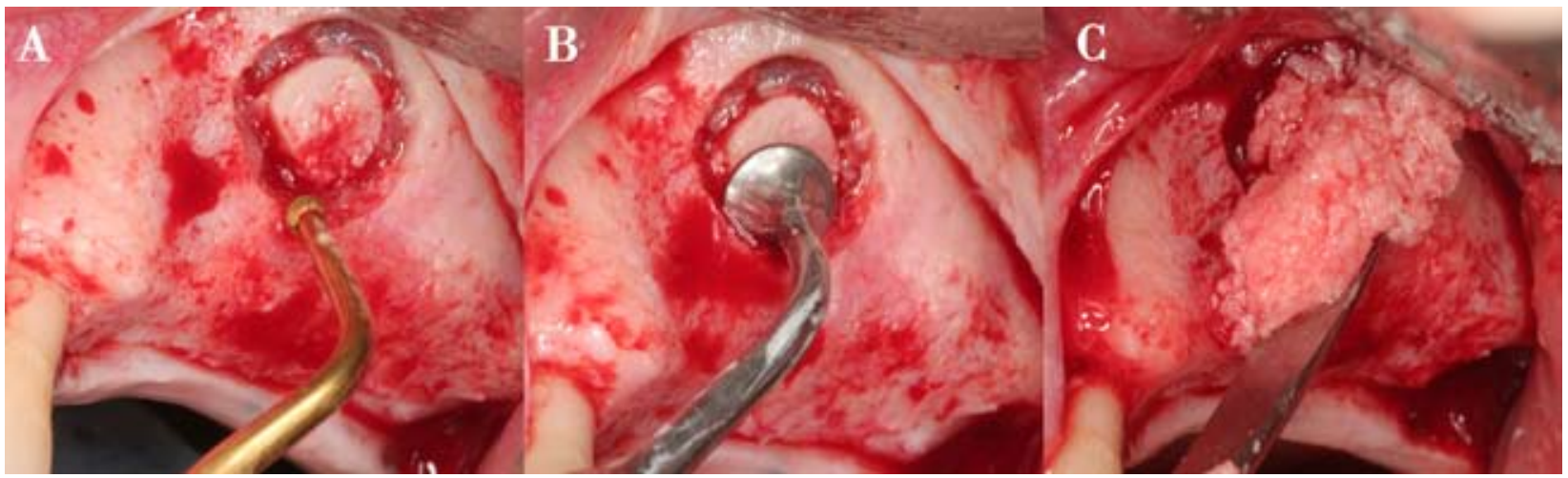

Fig. 1A. Active tips ES008 and ES008A of the Surgysonic ${ }^{\circledR}$ sy stem (Esacrom, Imola, Italy) were used to form an osseous window. Fig. 1B. The Schneiderian membrane was separated using tips ES 003A and ES003B.

Fig. 1C. The space was filled with graft material. 
The mucoperiosteal flap was closed primarily over the graft using 3-0 sutures (Lorca Marín, Murcia, España). A panoramic radiograph was taken following surgery. Antibiotics effective against both aerobic and anaerobic organisms were administered postoperatively for 7 days (Amoxicillin $500 \mathrm{mg} 3$ times a day) (8) (in case of allergy to penicillin, clindamycin $300 \mathrm{mg} 3$ times a day was administered). In addition, Ibuprofeno $600 \mathrm{mg} 3$ times a day (Bexistar ${ }^{\circledR}$, Laboratorio Bacino, Barcelona, España) was prescribed and patients were instructed to rinse with chlorhexidine $0.12 \%$ concentration (GUM®, John O. Butler CO, A Sunstar Company, Chicago, USA) 3 times a day.

The bone height obtained with the sinus lift was calculated in the postoperative orthopantomograph. The distortion was calculated, taking the implant length as a reference to compensate for the image extension that occurs in the orthopantomograph (Fig. 2). The bony edges of the maxillary alveolar process and the maxillary sinus floor were marked, and the distance between the two measured on the vertical implant axis (a). After the graft, the new maxillary sinus floor was delimited and the new distance to the bony edge of the maxillary alveolar process was measured again on the vertical implant axis (b). The difference between both measurements (b-a) determined bone gain.

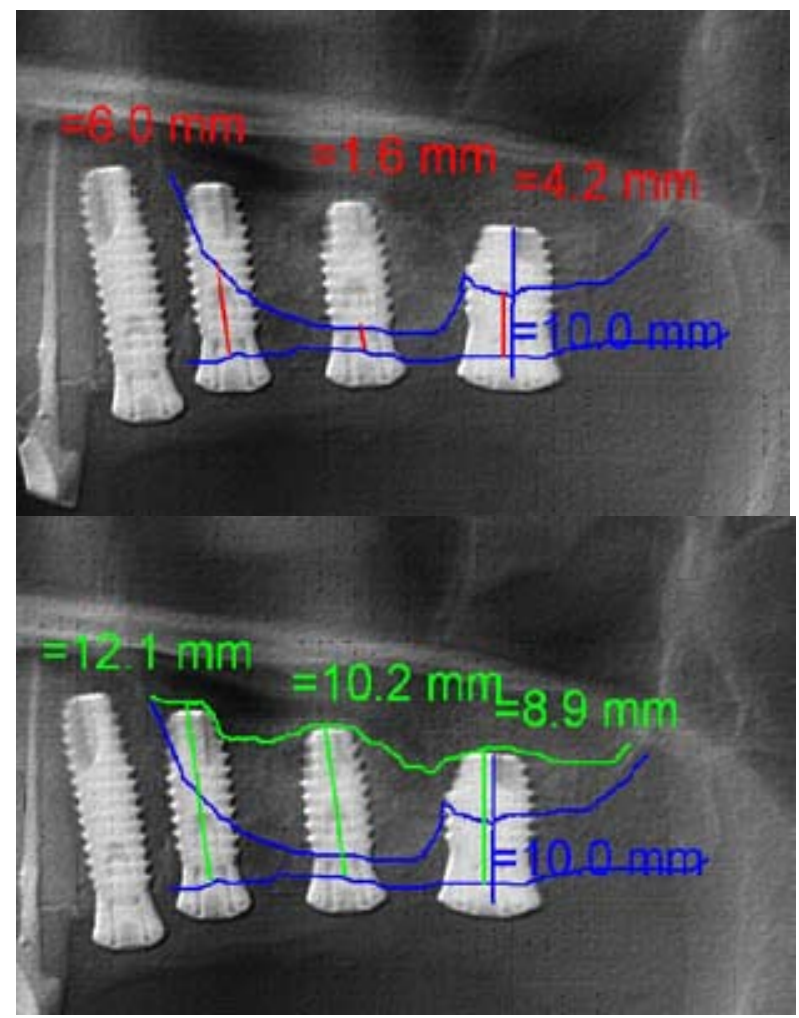

Fig. 2. The bone height obtained with the sinus lift was calculated in the postoperative orthopantomograph.

\section{Results}

A total of 26 sinus lift procedures were performed in 21 patients, 10 women and 11 men, with an average age of 49.9 years (range 29 - 66 years).

With respect to tobacco use, 15 patients were non smokers, 4 smoked 1-10 cigarettes a day and one patient more than 20 cigarettes a day.

In 14 sinus lift procedures implants were placed simultaneously and in 12 sinus lift implants were placed at a second intervention.

Of the 26 maxillary sinus lift, 16 were unilateral and 5 were bilateral, 9 were in the first quadrant and 17 in the second; 48 implants were placed. Four perforations of the Schneiderian membrane were observed, none of these were bilateral, 2 were in the first quadrant and 2 in the second quadrant. All perforations were less than $5 \mathrm{~mm}$, and so were patched with a collagen membrane in direct contact with the Schneiderian membrane. In these surgeries, 8 implants were placed. No patient had postoperative complications.

The average bone height prior to the intervention was $3.5 \mathrm{~mm}$ (range $0.6-8.7 \mathrm{~mm}$ ) and the average postsurgical bone height was $10.8 \mathrm{~mm}$ (range 7.5-15.6 mm). An average bone gain of $7.2 \mathrm{~mm}$ (range $2.5-11.7 \mathrm{~mm}$ ) was observed.

\section{Discussion}

In this study, sinus membrane perforations occurring during sinus lift procedures using ultrasound were evaluated, and the amount of bone gain was calculated.

The results indicate that with the use of the ultrasound in maxillary sinus lift $15.3 \%$ of membrane perforations occur, with an average bone gain of $7.2 \mathrm{~mm}$ (range 2.5$11.7 \mathrm{~mm}$ ).

The application of odontologic ultrasonic generators in the ostectomy of the bone window offers some advantages over conventional sinus augmentation surgery: the risk of perforating the Schneiderian membrane is reduced, improved view and hygiene of the operative area during ostectomy is achieved, and creation of a thinner and more conservative osseous incision is facilitated (3).

However, it should be noted that soft tissue structures, such as the mucous membrane of the maxillary sinus, may be perforated or injured by excessive mechanical force from the instrument tip (5).

Thor et al. (9) carried out 27 maxillary sinus lift (in 20 patients) with the conventional technique; there were 11 Schneiderian membrane perforations ( $41 \%$ of surgeries). Using the same technique, Schwartz-Arad et al. (4) observed similar results with 36 perforations (44\% of surgeries) in81 maxillary sinus lift. With ultrasound, Vercellotti et al. (6), in 15 patients, performed 21 maxillary sinus lift, finding a very low perforation percentage $(5 \%$ of surgeries); in our study, there were 4 membrane sinus 
perforations, representing $15.3 \%$ of the procedures. With respect to bone gain, Vercellotti (10) compared osseous repair (56 days after surgery) after using ultrasound and conventional burs; these authors observed that ultrasound provided a more favourable osseous repair. However, the average bone gain obtained in our study of 48 implants (7.2 $\mathrm{mm}$ average bone gain) was similar to results obtained by Thor et al. (9) $(6.51 \mathrm{~mm}$ average bone gain) using the conventional technique. Based on the results of this study, in ultrasound sinus lift procedures there are few Schneiderian membrane perforations, all small in size.

\section{References}

1. Barone A, Santini S, Sbordone L, Crespi R, Covani U. A clinical study of the outcomes and complications associated with maxillary sinus augmentation. Int J Oral Maxillofac Implants. 2006;21:81-5.

2. Sorní M, Guarinós J, García O, Peñarrocha M. Implant rehabilitation of the atrophic upper jaw: a review of the literature since 1999. Med Oral Patol Oral Cir Bucal. 2005;10 Suppl 1:E45-56.

3. Torrella F, Pitarch J, Cabanes G, Anitua E. Ultrasonic ostectomy for the surgical approach of the maxillary sinus: a technical note. Int J Oral Maxillofac Implants. 1998;13:697-700.
4. Schwartz-Arad D, Herzberg R, Dolev E. The prevalence of surgical complications of the sinus graft procedure and their impact on implant survival. J Periodontol. 2004;75:511-6.

5. Stübinger S, Kuttenberger J, Filippi A, Sader R, Zeilhofer HF. Intraoral piezosurgery: preliminary results of a new technique. J Oral Maxillofac Surg. 2005;63:1283-7.

6. Vercellotti T, De Paoli S, Nevins M. The piezoelectric bony window osteotomy and sinus membrane elevation: introduction of a new technique for simplification of the sinus augmentation procedure. Int J Periodontics Restorative Dent. 2001;21:561-7.

7. Hernández-Alfaro F, Torradeflot MM, Marti C. Prevalence and management of Schneiderian membrane perforations during sinuslift procedures. Clin Oral Implants Res. 2008;19:91-8.

8. Peleg M, Mazor Z, Garg AK. Augmentation grafting of the maxillary sinus and simultaneous implant placement in patients with 3 to 5 $\mathrm{mm}$ of residual alveolar bone height. Int J Oral Maxillofac Implants. 1999;14:549-56.

9. Thor A, Sennerby L, Hirsch JM, Rasmusson L. Bone formation at the maxillary sinus floor following simultaneous elevation of the mucosal lining and implant installation without graft material: an evaluation of 20 patients treated with 44 Astra Tech implants. J Oral Maxillofac Surg. 2007;65:64-72.

10. Vercellotti T, Nevins ML, Kim DM, Nevins M, Wada K, Schenk $\mathrm{RK}$, et al. Osseous response following resective therapy with piezosurgery. Int J Periodontics Restorative Dent. 2005;25:543-9. 